\title{
Exclusion of active Mycobacterium tuberculosis complex infection with the T-SPOT $^{\mathrm{TM}}$.TB assay
}

\author{
R.M.L. van Leeuwen*, A.W.J. Bossink" and S.F.T. Thijsen
}

\begin{abstract}
The present brief report describes four cases with mycobacterial infection and negative T-SPOT ${ }^{\mathrm{TM}}$.TB tests. This test proved to be a useful tool to help rule out the diagnosis of active Mycobacterium tuberculosis infection and could therefore prevent unnecessary or inappropriate therapy.
\end{abstract}

KEYWORDS: ELISPOT assay, interferon- $\gamma$-releasing assay, nontuberculous mycobacteria, tuberculin skin test, tuberculosis

$\mathbf{T}$ uberculosis (TB) is a major health problem with 8 million people infected annually and 2-3 million deaths each year throughout the world [1]. Even in low TB endemic countries, such as the Netherlands, clinicians are often confronted with patients suspected of mycobacterial infection. Diagnosis of TB can be difficult and combines clinical presentation, radiological results, acid-fast staining, molecular tests and culture. Definitive diagnosis is based on culture of a mycobacterium of the Mycobacterium tuberculosis complex. In the Netherlands, only $70 \%$ of presumed cases of pulmonary TB receiving treatment are culture confirmed [2]. Since cultures have only a limited sensitivity and clinical isolates can be very difficult to obtain, intensive clinically validated tests which detect the DNA of $M$. tuberculosis complex are accepted as proof of TB infection. Molecular tests, such as PCR, have very high sensitivity but are subject to possible contamination and subsequent falsepositive results [3]. Even under the strictest PCR regimes, cross-contamination is always a genuine hazard leading to intense control experiment settings, which are often easily discarded to improve output from a molecular unit. Even with the use of molecular tests, PCR results can remain negative in proven $\mathrm{TB}$ patients due to sampling errors.

In light of these diagnostic problems, clinicians often rely on tuberculin skin testing (TST) to demonstrate an infection with $M$. tuberculosis. However, the diagnostic value of TST is limited in several groups of patients. In immunocompromised patients, TST might produce false negatives due to anergy, bacille Calmette-Guérin (BCG)-vaccinated patients cannot be evaluated with TST due to the inherent cross-reactivity of purified protein derivate from the BCG strain used for vaccination, and in elderly patients the likelihood of a positive TST is relatively high due to the previous infectious burden [4]. TST can also give false-positive results in patients infected with environmental mycobacteria, such as $M$. avium complex, thereby limiting the use of TST in clinical decision making.

Treatment of TB is associated with moderate-tosevere side-effects, including polyneuropathy and hepatitis [5]. It is therefore important to correctly diagnose TB before the onset of therapy. Recently, two new tests for the diagnosis of TB infection have become commercially available. Both tests use the early secretory antigenic target6 and the culture filtrate protein- 10 peptides to stimulate $M$. tuberculosis-sensitised T-cells to produce interferon (IFN)- $\gamma$. The QuantiFERONTB Gold (Cellestis Ltd, Victoria, Australia) uses an ELISA technique for detecting IFN- $\gamma$ levels and the T-SPOT ${ }^{\mathrm{TM}}$.TB (Oxford Immunotec, Oxford, UK) uses an enzyme-linked immunospot (ELISPOT) technique to detect IFN- $\gamma$-producing T-cells [6]. The majority of mycobacteria other than $M$. tuberculosis complex lack these specific peptides making the tests highly specific [7-9], with cross-reactivity documented for $M$. marinum, M. kansasii and M. szulgai only [10].
AFFILIATIONS

Depts of *Internal Medicine,

\#Pulmonology, and

"Microbiology and Immunology, Diakonessenhuis, Utrecht, The Netherlands.

\section{CORRESPONDENCE}

A.W.J. Bossink

Bosboomstraat 1

3582 KE Utrecht

The Netherlands

Fax: 31302566738

E-mail: aikbossink@mac.com

Received:

May 142006

Accepted after revision:

July 142006

SUPPORT STATEMENT

A.W.J. Bossink is an endorsed member of the advisory board of Oxford Immunotec Ltd, Oxford, UK.

STATEMENT OF INTEREST

None declared.

European Respiratory Journal Print ISSN 0903-1936 Online ISSN 1399-3003 
The present report describes four patients with mycobacterial disease and suspected active TB in which the T-SPOT ${ }^{\mathrm{TM}} . \mathrm{TB}$ made the presence of TB infection unlikely.

\section{CASE REPORTS}

Case A was an 18-yr-old female Bosnian refugee, with a medical history of asthma, who presented with severe respiratory distress and fever. Her respiratory rate was 24 beats $\cdot \mathrm{min}^{-1}$ using her accessory muscles. On auscultation, a prolonged wheezing expiration was noticed. Blood tests showed an elevation of infectious disease parameters and hypoxaemia. Chest radiography showed an infiltrate and volume loss of the pulmonary lingula.

The patient was taken to the pulmonary ward and treated with ceftriaxone for supposed community-acquired pneumonia and subsequent worsening of her asthma. Volume loss of the left lung continued, she underwent a bronchoscopy and bronchoalveolar lavage (BAL) fluid was obtained. The smear was positive for acid-fast bacilli. PCR for $M$. tuberculosis complex (COBAS AMPLICOR PCR; Roche Diagnostics, Woerden, the Netherlands) was negative and was available 7 days after the smear result. Culture of the BAL fluid remained negative for mycobacteria.

In the absence of a BCG scar, a TST was carried out and was found to be positive ( $26 \mathrm{~mm}$ induration). Later, the father of the patient indicated that she had received a BCG vaccination during her childhood. A T-SPOT ${ }^{\mathrm{TM}}$.TB test result was negative. The patient did not receive any further antimicrobial treatment and recovered spontaneously. At 10 months' follow-up no signs of TB were apparent.

Case B was a 54-yr-old male with a medical history of chronic obstructive pulmonary disease (COPD) and interstitial nephritis, for which he was treated with prednisone and azathioprine during the previous 12 months. He was considered to be immunocompromised and was admitted to hospital when his COPD worsened.

Chest radiography showed diffuse densities in both lungs and high-resolution computed tomography scan of the chest showed nodular lesions and ground-glass attenuation. Examination of the BAL showed the presence of acid-fast bacilli and the patient was put in aerogenic isolation. A TST was not performed and the T-SPOT ${ }^{\mathrm{TM}}$.TB test result was negative. PCR for $M$. tuberculosis complex was negative and available 12 days after the smear result. Cultures were positive for M. genavense.

Case C was a 66-yr-old male who produced purulent sputum for a few months. There were no complaints of fever, haemoptysis or weight loss. Chest radiography and computed tomography demonstrated a noncavitating lesion in the apical segment of the left lower lobe and enlarged mediastinal lymph nodes. Laboratory results showed raised infectious parameters. A positron emission tomography scan showed moderately increased activity in the lesion and in two mediastinal lymph nodes. A T-SPOT ${ }^{\mathrm{TM}}$.TB test result was negative. Culture of the BAL fluid turned positive with mycobacteria after 5 days. PCR for $M$. tuberculosis complex was negative and available 6 days after the BAL. Definitive diagnosis was obtained 10 days after lavage as the mycobacteria were determined as M. avium.
Transthoracic fine needle aspiration (T-FNA) to exclude malignancy produced acid-fast bacilli from which $M$. avium was again cultured; no malignancy was found. There were no indications that the patient was immunocompromised. After treatment with ethambutol and clarithromycin, the lesion in the left lobe disappeared and the patient recovered.

Case D was a 75-yr-old female smoker who presented to the outpatient clinic with symptoms of coughing and expectoration of white sputum. She experienced weight loss and tiredness. There was no history of haemoptysis. On physical examination, no abnormalities were found. Chest radiography and computed tomography showed a cavitating lesion. The erythrocyte sedimentation rate was slightly raised and TST and $\mathrm{T}_{-} \mathrm{SPOT}^{\mathrm{TM}}$.TB test results were negative.

Bronchoscopy showed viscous secretion in the bronchi. Culture of BAL fluid remained negative. T-FNA was analysed by the pathologist and showed granulomatous inflammation with necrosis and acid-fast bacilli. PCR for $M$. tuberculosis complex was negative and available 29 days after the pathology result. Finally, the T-FNA culture grew M. malmoense. After treatment with ethambutol, rifampicin and clarithromycin for 9 months, the lesion resolved.

\section{DISCUSSION}

Clinical presentations varied in the four patients but all had acid-fast bacilli in specimens obtained from their lungs; therefore, a diagnosis of TB was considered. TST was performed in two patients, one with misleading results, probably due to previous BCG vaccination. When TB is suspected, it results in infection-control measures, contact investigation via the public health service and the start of treatment with tuberculostatics with inherent side-effects. Speed to rule out TB is of great importance to prevent unnecessary actions and distress. For that purpose, the COBAS AMPLICOR PCR was used for $M$. tuberculosis complex. However, several reports have shown that performance of such tests are limited; REISCHL et al. [11], in 1,149 patient samples, demonstrated a sensitivity of $84 \%$ as compared to culture, indicating that a negative PCR does not rule out TB. In the current study, PCR results were available after an average of 14 days. Since PCR was not available in the present authors' laboratory, the relatively long interval between initial diagnosis and PCR result probably reflects logistic problems, with many other smaller laboratories known to suffer the same problem. Theoretically, PCR results can be available very rapidly but, in clinical practice, PCR results can take a long time. Conversely, PCR can be run on stored materials and in theory on virtually any material, although the COBAS AMPLICOR PCR has only been validated for some respiratory specimens. Laboratory facilities for ELISPOT techniques are low profile and therefore $\mathrm{T}_{-} \mathrm{SPOT}^{\mathrm{TM}}$.TB could be easily implemented. Limitations in the use of $\mathrm{T}_{-} \mathrm{SPOT}^{\mathrm{TM}} . \mathrm{TB}$ are the relatively short time-frame allowed between blood sampling and processing. Furthermore, sample processing has room for error in separating, counting and incubation of peripheral blood mononuclear cells and counting the spot-forming units after completion of the test. To date, no comparative studies between the performance of ELISPOT and PCR have been published. 
Depending on the clinical setting, $\mathrm{T}^{-\mathrm{SPOT}^{\mathrm{TM}}}{ }^{\mathrm{T}} \mathrm{TB}$ has a sensitivity of $83-100 \%$ in active TB $[6,12,13]$. Over a period of $1 \mathrm{yr}, 19$ patients with active, culture-confirmed TB infection were diagnosed in the present authors' hospital (Diakonessenhuis Hospital, Utrecht, the Netherlands). All of these patients were T-SPOT ${ }^{\mathrm{TM}}$.TB positive with no indeterminate results observed (unpublished data). In an attempt to exclude $M$. tuberculosis infection, it seems reasonable to use historic M. tuberculosis culture and T-SPOT ${ }^{\mathrm{TM}} . \mathrm{TB}$ test results from the laboratory as a control for sensitivity for the detection of $M$. tuberculosis infection. Based on the high sensitivity in active disease, a negative test makes active TB unlikely. However, two recent papers [12, 13] demonstrated sensitivity for active disease of 83 and 97\%, respectively. Unfortunately, the paper by FERRARA et al. [13] did not mention whether the false-negative ELISPOT results concerned immunocompromised patients. An increase of indeterminate ELISPOT test results was reported in patients undergoing cancer chemotherapy, but not in young children, the elderly or patients receiving steroid therapy [13]. Specificity of an ELISPOT is difficult to calculate in patients with $M$. tuberculosis complex because a positive $\mathrm{T}-\mathrm{SPOT}^{\mathrm{TM}}$.TB result can also be the consequence of latent $\mathrm{TB}$ infection in combination with $M$. tuberculosis complex infection. To be able to calculate the specificity of the $\mathrm{T}_{-S P O T}{ }^{\mathrm{TM}} . \mathrm{TB}$ to rule out active $\mathrm{TB}$, the background prevalence of positive $\mathrm{T}-\mathrm{SPOT}^{\mathrm{TM}} . \mathrm{TB}$ results in a population must be known. These data are contrary to current data on TST results in the general population not available. However, MEIER et al. [12] report the specificity to rule out active TB at $92 \%$ or 11 out of 12 cases. Taking into account the small numbers in the present report, the specificity would be $100 \%$.

In case $\mathrm{A}$, laboratory cross-contamination cannot be excluded but is unlikely because the number of acid-fast bacilli seen on the direct stain was rather high. However, even if this case represented a laboratory cross-contamination, the negative $\mathrm{T}_{-S P O T}{ }^{\mathrm{TM}}$.TB test result would have prevented unnecessary therapy.

Apart from case B, who was suffering from disseminated $M$. tuberculosis complex infection, no patients described in the present report were likely to be immunocompromised. Using $\mathrm{T}^{-S P O T}{ }^{\mathrm{TM}}$.TB in immunocompromised patients to make active TB highly unlikely has to be performed with great caution as the available data suggests a decreased sensitivity for the ELISPOT of, for example, only $90 \%$ in HIV-positive patients [9]. Indeterminate test results cannot and should not be interpreted as a negative test result.

In the present report, the T-SPOT ${ }^{\mathrm{TM}} . \mathrm{TB}$ was used to make the possibility of active infection with $M$. tuberculosis complex very unlikely, avoiding unnecessary and inappropriate therapy [12]. Furthermore, a negative T-SPOT ${ }^{\mathrm{TM}}$.TB directs the microbiologist to PCR and culture for mycobacteria other than TB.

In conclusion, in low tuberculosis endemic settings, the $\mathrm{T}_{-\mathrm{SPOT}^{\mathrm{TM}}}{ }^{\mathrm{T}}$.TB test is a useful tool to help rule out the diagnosis of active tuberculosis in immunocompetent patients, as demonstrated in these patients, and therefore prevents unnecessary or inappropriate therapy.

\section{REFERENCES}

1 Dye C, Scheele S, Dolin P, Pathania V, Raviglione MC. Global burden of tuberculosis: estimated incidence, prevalence, and mortality by country. Consensus Statement: WHO Global Surveillance and Monitoring Project. JAMA 1999; 282: 677-686.

2 Anonymous. Royal Netherlands Tuberculosis Association (KNCV) Index Tuberculosis 2001-2002. ISDN90-77865-01-2. The Hague, 2004.

3 Kwok S, Higuchi R. Avoiding false positives with PCR. Nature 1989; 339: 237-238.

4 Borgdorff MW, van der Werf MJ, de Haas PE, Kremer K, van Soolingen D. Tuberculosis elimination in the Netherlands. Emerg Infect Dis 2005; 11: 597-602.

5 McNeill L, Allen M, Estrada C, Cook P. Pyrazinamide and rifampin $v s$ isoniazid for the treatment of latent tuberculosis: improved completion rates but more hepatotoxicity. Chest 2003; 123: 102-106.

6 Pai M, Riley LW, Colford JM Jr. Interferon- $\gamma$ assays in the immunodiagnosis of tuberculosis: a systematic review. Lancet Infect Dis 2004; 4: 761-776.

7 Lalvani A, Pathan AA, Durkan H, et al. Enhanced contact tracing and spatial tracking of Mycobacterium tuberculosis infection by enumeration of antigen-specific T cells. Lancet 2001; 357: 2017-2021.

8 Lalvani A, Pathan AA, McShane H, et al. Rapid detection of Mycobacterium tuberculosis infection by enumeration of antigen-specific T cells. Am J Respir Crit Care Med 2001; 163: 824-828.

9 Chapman A, Munkanta M, Wilkinson KA, et al. Rapid detection of active and latent tuberculosis infection in HIVpositive individuals by enumeration of Mycobacterium tuberculosis-specific T cells. AIDS 2002; 16: 2285-2293.

10 Arend S, van Meijgaarden KE, de Boer K, et al. Tuberculin skin testing and in vitro $\mathrm{T}$ cell responses to ESAT-6 and culture filtrate protein 10 after infection with Mycobacterium marinum or M. kansasii. J Infect Dis 2002; 186: 1797-1807.

11 Reischl U, Lehn N, Wolf H, Naumann L. Clinical evaluation of the automated COBAS AMPLICOR MTB assay for testing respiratory and nonrespiratory specimens. J Clin Microbiol 1998; 36: 2853-2860.

12 Meier T, Eulenbruch H-P, Wrighton-Smith P, Enders G, Regnath T. Sensitivity of a new commercial enzyme-linked immunospot assay (T SPOT-TB) for diagnosis of tuberculosis in clinical practice. Eur J Clin Microbiol Infect Dis 2005; 24: 529-536.

13 Ferrara G, Losi M, D'Amico R, et al. Use in routine clinical practice of two commercial blood tests for diagnosis of infection with Mycobacterium tuberculosis: a prospective study. Lancet 2006; 367: 1328-1334. 